\title{
NEW AIRBORNE SENSORS AND PLATFORMS FOR SOLVING SPECIFIC TASKS IN REMOTE SENSING
}

\author{
G. Kemper \\ Geotechnik, Geoinformatik \& Service GmbH, Speyer / Germany \\ kemper@ggs-speyer.de
}

KEY WORDS: Sensors, airborne, platform, GPS-IMU, aerial camera, Lidar, multispectral

\begin{abstract}
:
A huge number of small and medium sized sensors entered the market. Today's mid format sensors reach 80 MPix and allow to run projects of medium size, comparable with the first big format digital cameras about 6 years ago. New high quality lenses and new developments in the integration prepared the market for photogrammetric work. Companies as Phase One or Hasselblad and producers or integrators as Trimble, Optec, and others utilized these cameras for professional image production.

In combination with small camera stabilizers they can be used also in small aircraft and make the equipment small and easy transportable e.g. for rapid assessment purposes. The combination of different camera sensors enables multi or hyper-spectral installations e.g. useful for agricultural or environmental projects. Arrays of oblique viewing cameras are in the market as well, in many cases these are small and medium format sensors combined as rotating or shifting devices or just as a fixed setup. Beside the proper camera installation and integration, also the software that controls the hardware and guides the pilot has to solve much more tasks than a normal FMS did in the past. Small and relatively cheap Laser Scanners (e.g. Riegl) are in the market and a proper combination with MS Cameras and an integrated planning and navigation is a challenge that has been solved by different softwares. Turnkey solutions are available e.g. for monitoring power line corridors where taking images is just a part of the job. Integration of thermal camera systems with laser scanner and video capturing must be combined with specific information of the objects stored in a database and linked when approaching the navigation point.
\end{abstract}

\section{INTRODUCTION}

Existing technologies typically use three extreme platforms, satellites, aircrafts or terrestrial close range ones. Between the aerial and terrestrial use is a field for innovative applications, which are typically experimental but in some cases already well established and operational. It is possible to produce data in resolution and accuracy, which are ranking between the aerial and terrestrial techniques. Budget and administrative rules sometimes limit the use of aircrafts. Flexibility, accuracy and scientific interest are other reasons for such applications. In some cases these new technologies are able to compete with traditional ones and already have entered the market.

\section{BALLOON AND BLIMP PHOTOGRAMMETRY}

Transportability, costs efficiency and flexibility for the application in archaeology are main reasons to make use of balloons in the projects Patara (Turkey) and the Acropolis Athens (Greece). The goal in both cases was the image based photogrammetric analysis of historical objects, in Patara the old Lykian Theatre and in Athens the Acropolis.

The estimated GSD was defined between 0.2 and $2 \mathrm{~cm}$. For that, calibrated small or mid-format cameras were used and fixed below a Helium balloon. Like that it was moved with 3 ropes to the target position. In both applications a cardanical stabilisation below the HE balloon was used to keep the camera nadir viewing.

In „Patara“, a calibrated 4 MPix Olympus compact-camera was used and remotely controlled via a wire connection. Using a monitor transmitting the finders view, the operator was able to move the balloon with camera to the target point visually.

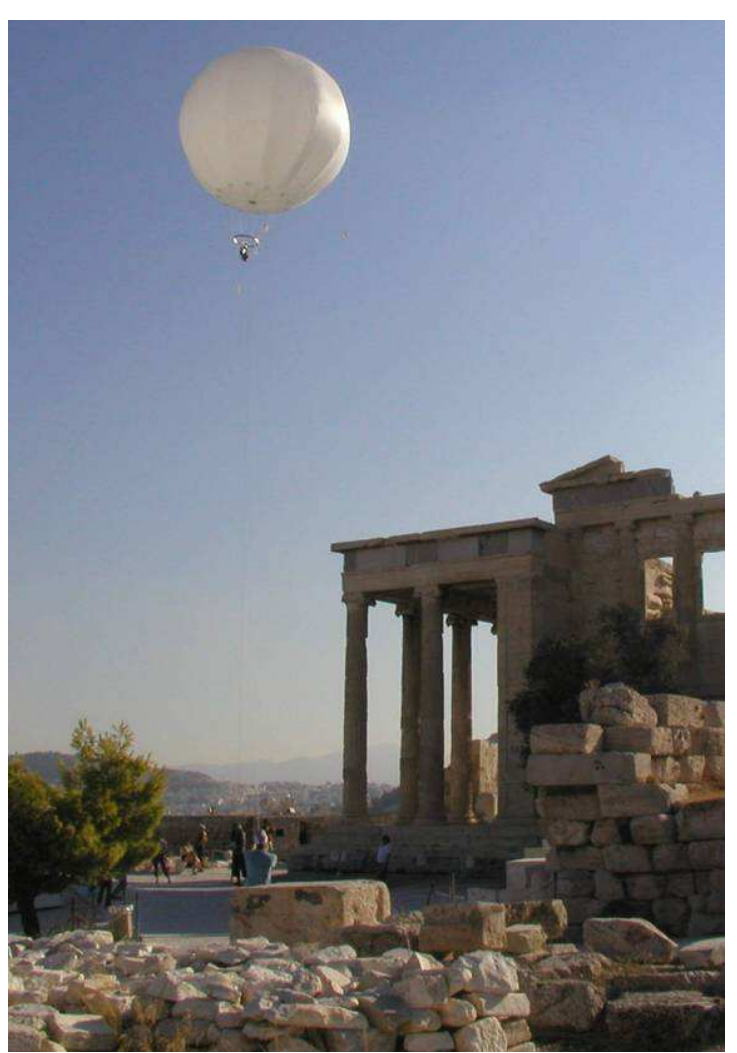

Figure 1: Balloon-photogrammetry for the Acropolis Athens in Greece 
The application on the Acropolis used of a Rollei Metric 6008 where an electronic release- and event-connection was implemented. A wide range blue tooth device transmitted the GPS coordinates of the camera to a PC with a modified Flight Management System (AeroTopoL). That way, camera and balloon were moved to the pre-planned positions using a moving map and then released via the PC. By feed back of the event-port, released images are marked on the map and the metadata stored in a text protocol. With an additional feature, the FMS was able to rotate the camera in order to keep the kappa angles similar to the planning. An additional monitor transferred the view of the camera to the PC.

For supporting a Ground-Truth-Station in Portugal, a radiometric scanner below a balloon continuously collected the surface temperature. The data communication was solved with a DECT-Modem. Moving the balloon over the test area produced profiles of the surface temperature to validate the accuracy of Thermal IR channels of satellites.

A fourth application was established by the University of Beijing. It was designed as a classical aerial imaging project to monitor and map the urban sprawl of Chinese cities. The University of Beijing develops and produces airships (HEBlimps) that are controlled remotely via radio transmitters. In such an airship, a stabilized camera-platform (AeroStab-1) and a Rollei Metric aerial camera (AIC P45) were used for a test project. The GPS-IMU data were transmitted via a boosted radio modem and used in AeroTopoL FMS. Artificial instruments and moving maps enabled the pilot to navigate on the line, the camera then was released automatically.

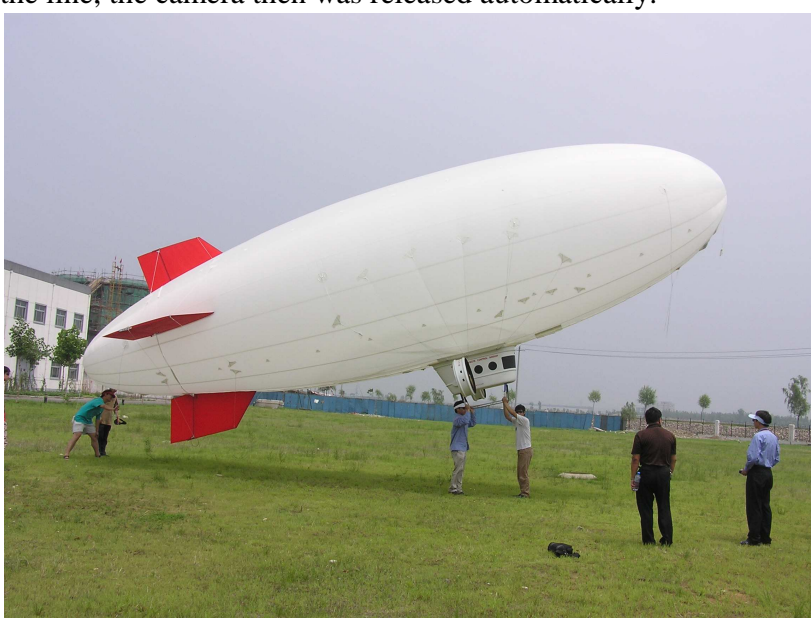

Figure 2: He-Blimp of the University Beijing with a stabilised platform as a carrier for a mid format aerial metric camera (Rollei AIC).

\section{TRIKES UND PARATRIKES}

The smallest and cheapest "aircrafts" are Trikes und Paratrikes. Paratrikes, also called motorized Paragliders consists of a parachute, a trike (exciter) and a rotax motor. They are available with 1 or 2 seats. Such an aircraft was carrier for an archaeological application using a SLR camera, a Laser scanner and a direct referencing device. A terrestrial Laser scanner from Riegl, a Nikon camera and AeroDiDOS for direct referencing (precise GPS-IMU) was interfaced. AeroTopol as mission planning, navigation and control software was used then and triggered both, Laserscanner and Camera simultaneously.

The next type of aircraft are so called Trikes, they consist of a delta wing, a trike and a Rotax motor. Most of them are 2 seaters.

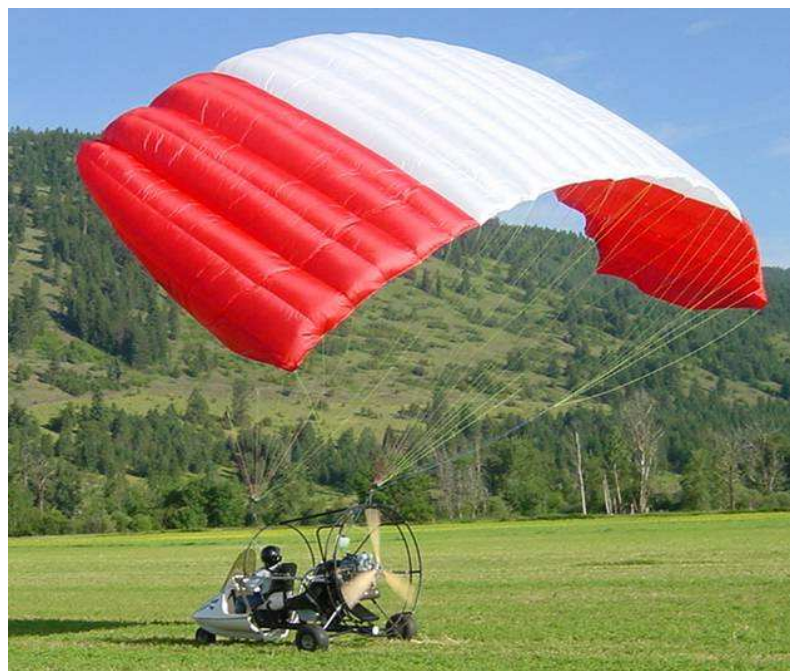

Figure 3: a paratrike with an exciter as a carrier system for airborne Lidar

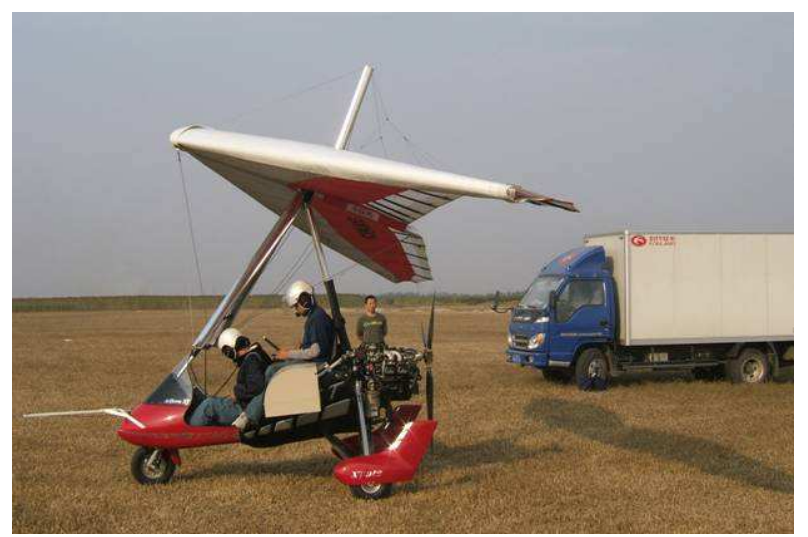

Figure 4: Trike of the Bureau for Surveying and Mapping of the Province Hebei / China

Already in operational use is such an aircraft at the Bureau for Surveying and Mapping of the Province Hebei / China. A Rollei AIC 39 MPix camera together with the gyro stabilised platform AeroStab-2 are its main components. Also here, AeroTopoL as Mission Planning and Flight Management Software is used. Since 2006, this regional administration performs regular missions to map and monitor the city development. In 2008, this system was also used to observe the earthquake area of Sichuan to monitor the destroyed buildings and infrastructure because this system is easy to transport, needs short runways and quickly is operational.

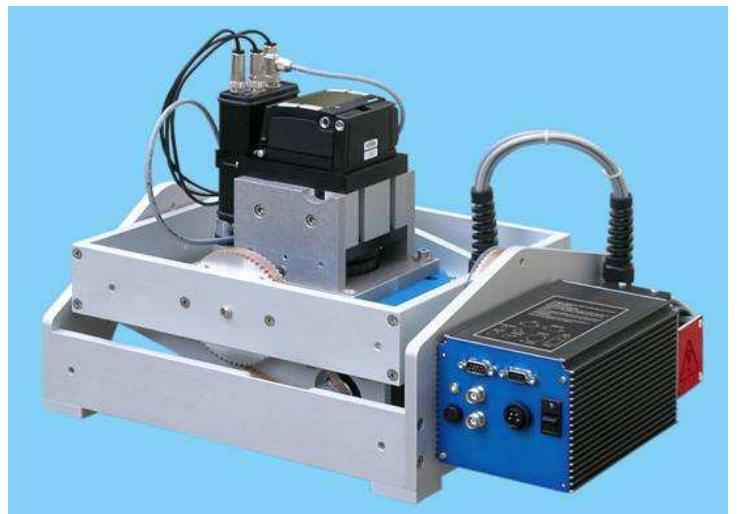

Figure 5: mid-format camera on the gyro stabilised platform AeroStab-2 
These missions are done with pilot and operator, analogue to classic aerial surveys using mission planning and target navigation.

The results of the gyro-stabilized platform are not very accurate (X, Y, Z 1-2 m, Roll, Pitch, Heading 0.5-1 degree), but with a $10 \mathrm{~Hz}$ refresh rate smooth for the navigation and precise enough for an automated tie-point matching with attached aerotriangulation All known methods like stereo-evaluation for 3D feature extraction, autocorrelation to derive 3D points for creating a DTM, ortho-rectification, orthophoto mosaicing and True-Orthophoto calculation are done in this state organisation. On one side big scaled images even without FMC can be captured since the minimum speed is less than $50 \mathrm{~km} / \mathrm{h}$. On the other hand the movement of the trike like a pendulum makes the stabilisation mandatory.

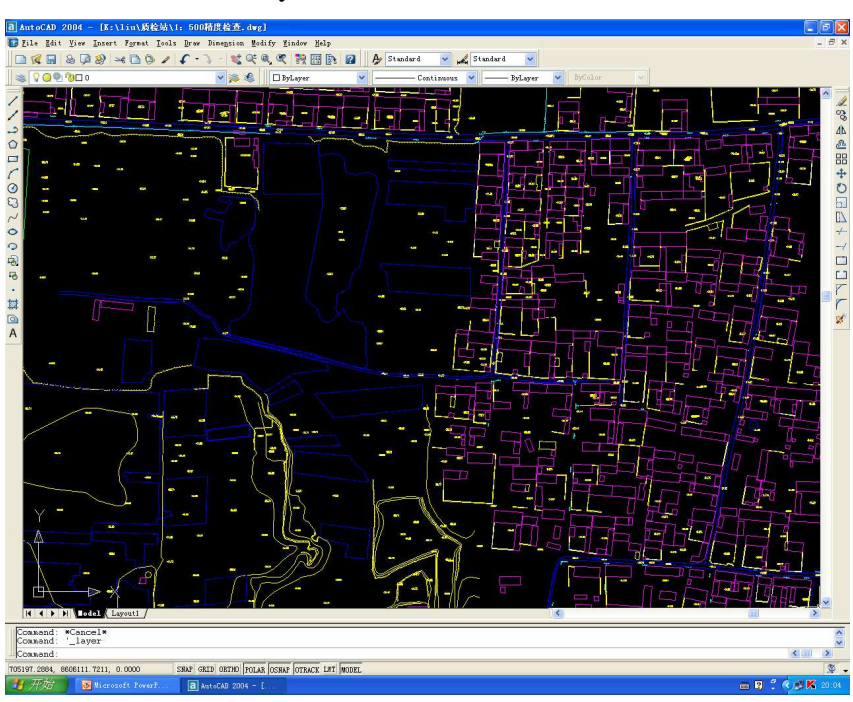

Figure 6: digital map in scale 1:2.000 based on a GSD of $10 \mathrm{~cm}$ produced with a Trike

\section{EXPERIMENTAL UND ULTRA LIGHT AIRCRAFTS}

There is a big variety of such planes, starting from simple self made aircrafts up to high technological planes equal in performance and technology to eco-class ones. Many small companies make use of such planes since modifications and changes are easy to do. Such an UL was designed by a Chinese company and already modified for aerial survey.

Between pilot and co-pilot in the back, a hole to host a small stabilized platform with camera was constructed. Like that, the operator has the entire equipment in view while the pilot receives all needed mission information via a pilot screen that is connected to a PC with the FMS.

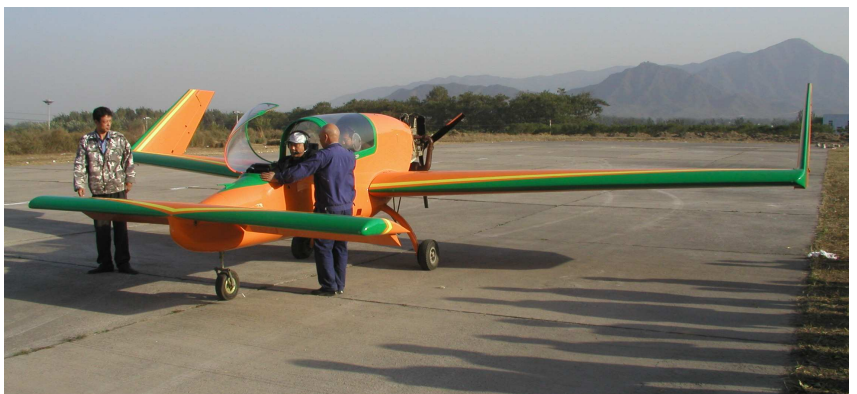

Figure 7: design of a „Duckwing“ ultra light aircraft of a Chinese company, ready to use for aerial survey

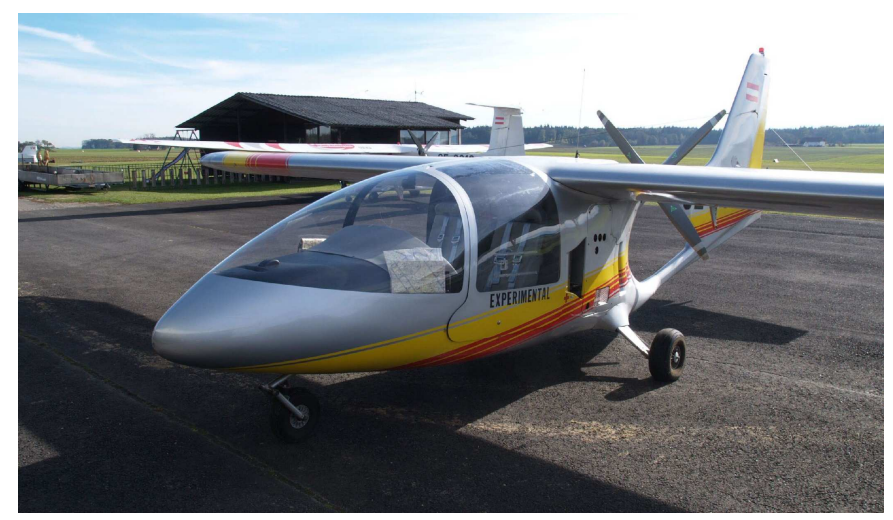

Figure 8: Experimental aircraft of the company Pauly Luftbildfotographie. Camera and stabilizer was mounted outside in a carbon box

Important at these aircraft league are the parameters for flying a proper mission. Some of them only in faster speeds fly stabile on the flight lines. That limits achieving big high resolution data.

Aircraft with up-wings and high wing profiles can fly slow and stabile and can precisely match the target points on the line. Like that, the company Pauly and its experimental aircraft was used for several test missions in cooperation with the University of Düsseldorf.

On the airport „Dahlemer Binz“ a calibration field with marked GCPs was designed and mission up to $5 \mathrm{~cm}$ GSD were performed successfully.

\section{CLASSICAL AIRCRAFTS, THEIR PLATFORMS AND SENSORS}

One of the most frequently used planes are the Cessna 175, 206 und 210, which can be easily found all over the world, You easily can charter them and some are already modified with a hole in the floor for aerial missions. Solutions have been found to enable the nadir view of the sensor even without hole,

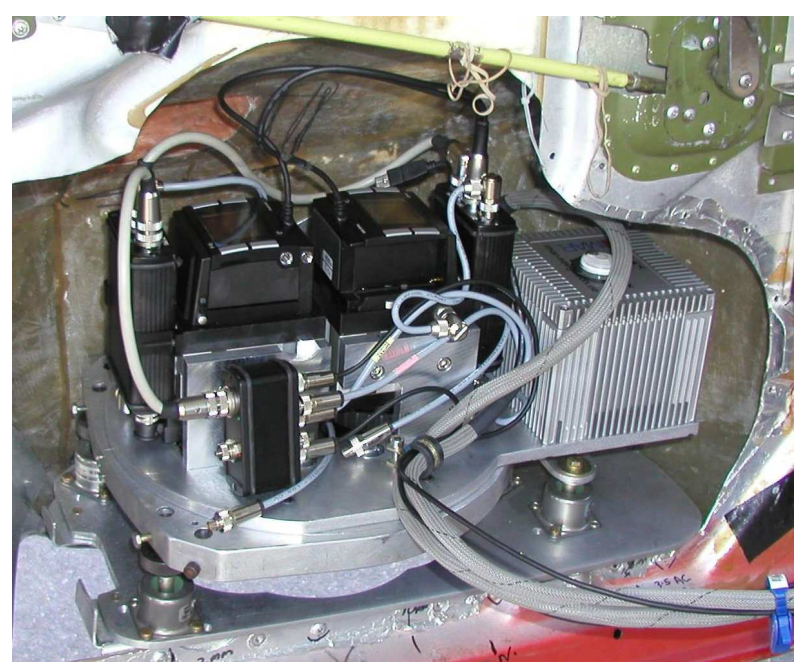

Figure 9: Installation of 2 mid-format cameras and AeroDiDOS (here FSAS IMU) for direct referencing in a modified door of a Cessna 210 
One example is a 2 camera installation for capturing RGB and NIR images simultaneously. In addition, this setup has a direct referencing using a FOG IMU installed in a "bump" mounted in the pilots door. This application is used for river monitoring to map blue and green algae with respect of irrigation and drinking water production in Australia.

Corridor mapping needs a relative high number of GCPs, not sufficient for such a monitoring. With the precise AeroDiDOS system, the projection centres were determined via ppp in $20 \mathrm{~cm}$ accuracy and the rotations in a few millidegree resolutions.

Both cameras (Rollei AIC P45 IR and RGB) are mounted together with FSAS IMU on a turntable for manual drift compensation in the carbon „bumb“. The influence on the aerodynamic in case of a Cessna 210 is low.

AeroDiDOS, AeroTopoL and Inertial explorer deliver precise results to start a nearly fully automated data processing to achieve CIR Orthophotos. Using standard AT procedures with tie point matching can increase the accuracy but this was not needed for this monitoring purpose.

A different solution was found for a Cessna 210 in Bolivia that was used for Coca-Monitoring. The luggage door was completely removed and the camera with stabilizer mounted outside the aircraft in a housing. Flying high with a long focus length ,supported the security“ for this mission.

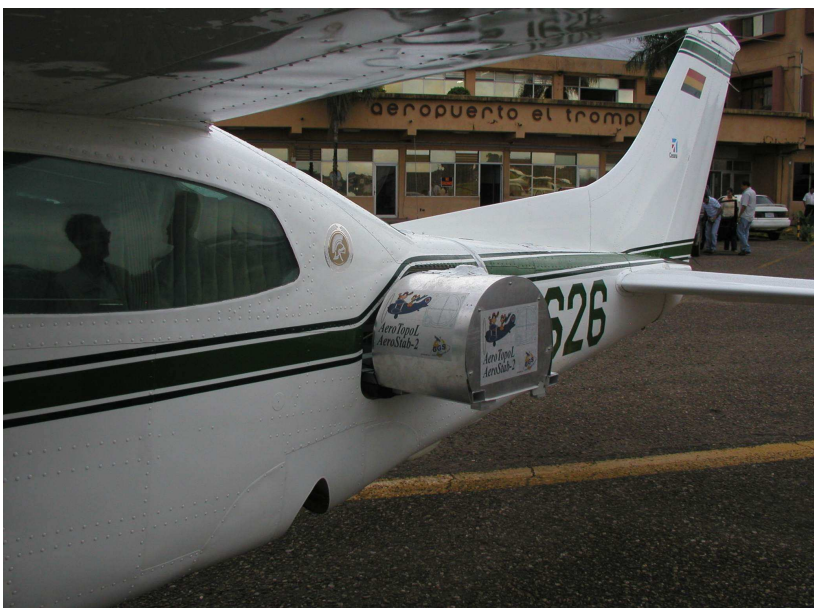

Figure 10: external mounted camera box in Bolivia, used for monitoring Coca plants

\section{THERMOGRAPHY}

The company Jenoptic is known for its experience in aerial cameras. The also produce thermal infrared cameras designed for aerial survey, but typically the are made for industrial or military applications.

The OEM sensor module was finalised to an AeroTherm Camera that can use different lenses. This camera was nicely integrated into the stabilized platform AeroStab-3 and the FMS AeroTopoL.

Thermal cameras operate in the frequency of the thermal IR spectrum and observe heat radiation. Beside that, also secondary effects as evaporation, heat buffering, water pollution and others can be interpreted from the data.

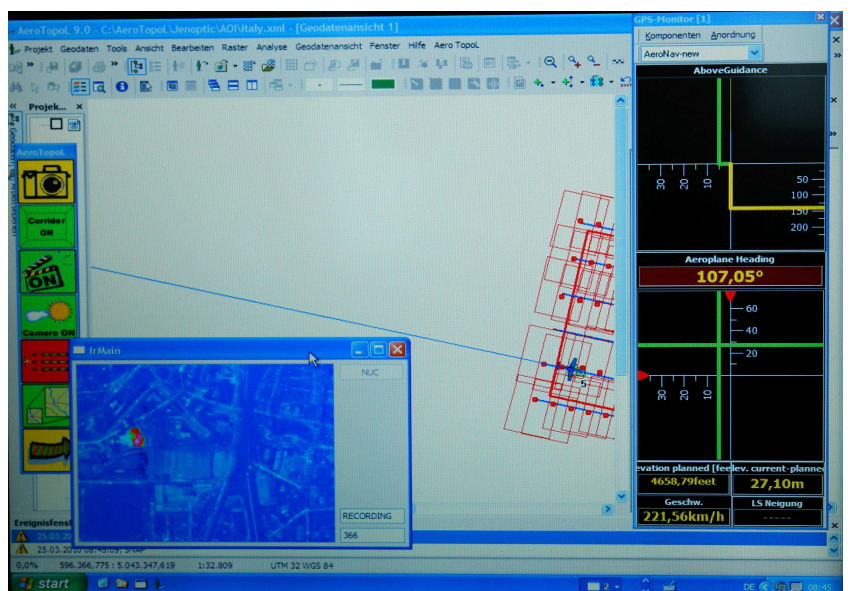

Figure 11: FMS AeroTopoL and the capture software IR Main with a live-view

There exist many applications for this camera today, beside the classical application to monitor buildings and their heat loss, applications in agriculture, ecology, hydrology and hydrography to monitor water flow and many more. Thermal cameras unfortunately have a very low geometric resolution $(640 * 480)$. Even a resolution of 0.3 to $1 \mathrm{~m}$ GSD is sufficient, wider areas need a huge number of images to be properly covered. They have also to be processed to a mosaic.

Most thermal cameras operate as a video camera this means that the FMS (AeroTopoL) just triggers on the relevant position to take one image out from the data stream. The Camera has its own software package to capture and handle the camera via fire wire. Both programs, the FMS AeroToopoL and the capture software IR Main run on the same PC and communicate via TCP/IP.

AeroTopoL creates a folder fort he images, sends beside the trigger signal a recommended name and the IR-Main copies the latest image of the data stream into that predefined folder. AeroTopoL takes an event-signal from the stabilizer and waits for a report that IR Main has stored the image under the certain name. This is then documented in the AeroTopoL Report files. For each line, AeroTopoL forces the IR Main Software to do a radiometric calibration of the camera. This can be also done manually as well as the focusing of the lens.

The stored orientation data from the FMS are suitable to start a automatic tie point matching of the image block. The small size of the images and the 16 bit absolute grey value makes this procedure not simple. Nevertheless, using adapted algorithms (e.g. to grey balance the entire image block and to find feature based tie points with PhoTopoL Software), automatic processing of the data is possible since urgently needed for projects due to the big number of images.

Other developments will be the combination of 2 or 3 cameras in combination with direct referencing equipment (AeroDiDOS). That way, the needed number of flight lines can be reduced and the processing of data be optimized. Active cooled cameras might assist in drift free radiometry especially for observing small temperature differences (water, agriculture...) 


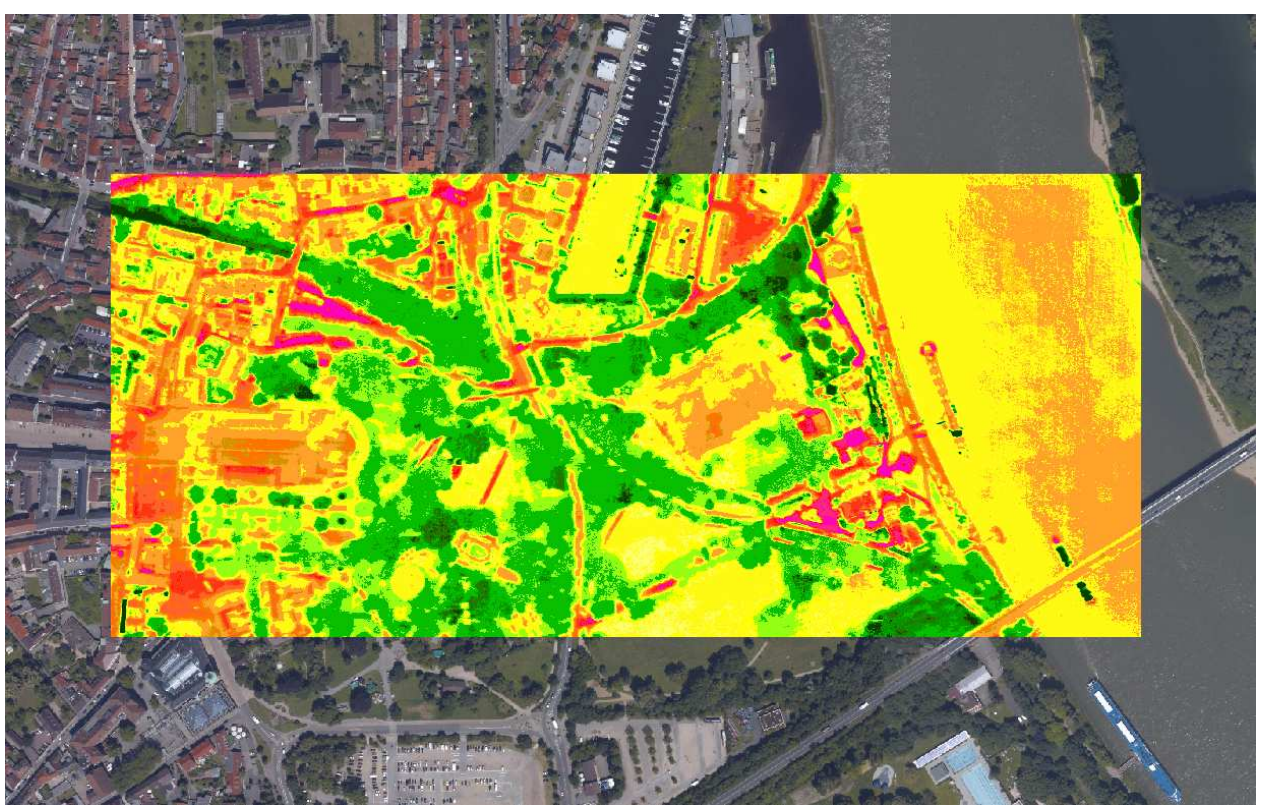

Figure 12: Example of a ThIR Orthomosaic overlaid on an Orthophoto of a test site in Speyer

\section{MULTISPEKTRAL APPLICATION - AIRAGRO}

A rather new application was designed in a combination of a mid format and 2 SLR Cameras.

The projects goal was the airborne monitoring of agricultural areas. Good priced technical components, cost-effective carrier systems and automated data processing are the project targets. The midformat camera (Rollei P25) captured the RGB Image as master and the 2 SLR cameras (Nikon D700) were modified to capture 2 different NIR channels in order to cover the „red edge“. The projection centres were measured with a Septendrio L1/L2 receiver and a MEMs based IMU that was loosely coupled with the GPS to determine the rotation angles. Applying Offsets and misalignment values, the result was convenient to reduce GCPs significantly. The RGB images were processed by standard photogrammetric procedures and the IR data co-registered to them.

Figure 13: Integration scheme of the technical components of the Project AirAgro
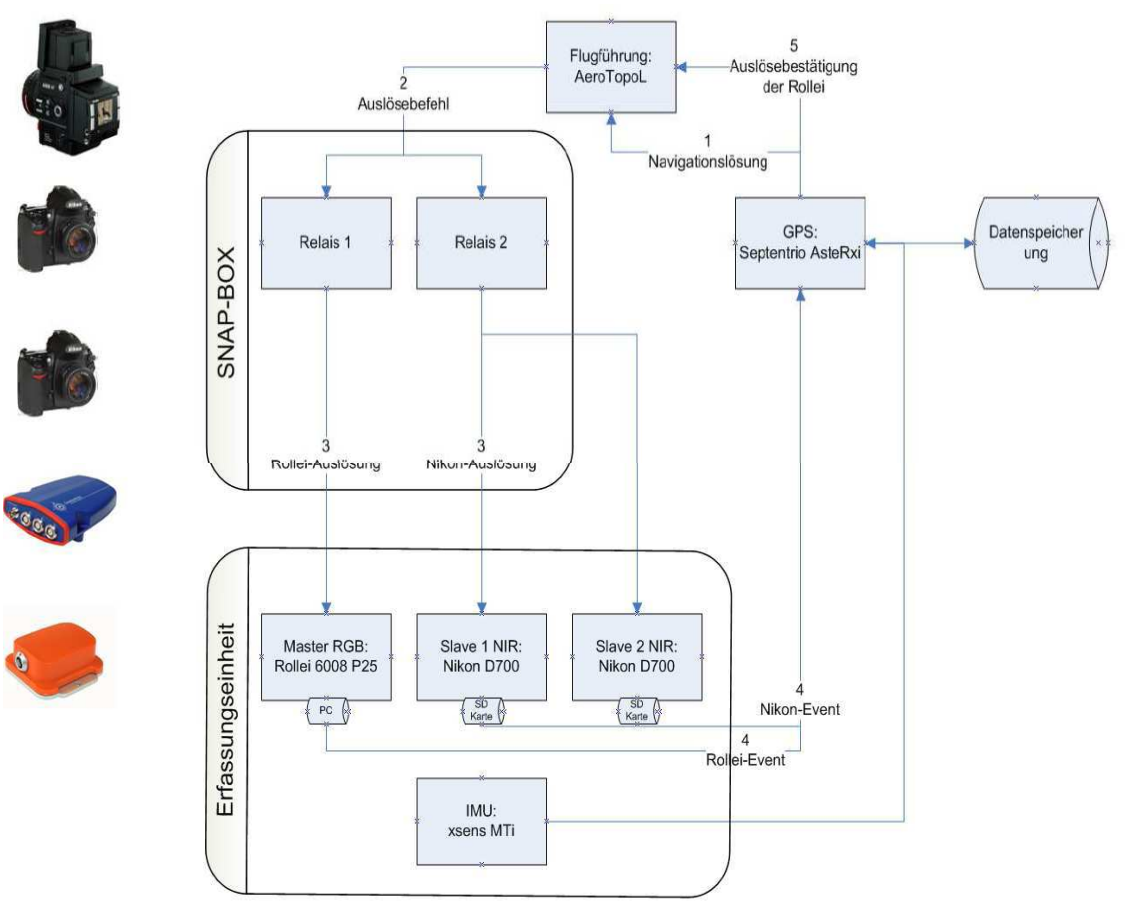


\section{LITERATURE}

KEMPER, G., ALTAN, O., CELIKOYAN, M., TOZ, G., (2003): Ballonluftbild- und GIS-basierte photogrammetrische Auswertung kulturhistorischer Objekte in Patara / Türkei. Angewandte Geographische Informationsverarbeitung IX - Agit 2003, Wichmann, Heidelberg

KEMPER, G., (2006): New airborne Sensors and Platforms for solving experimental Applications in Photogrammetry and Remote Sensing. Fifth International Symposium "TurkishGerman Joint Geodetic Days", Berlin.

KEMPER, G. (2006): Spezialapplikationen in der luftgestützten Fernerkundung- Preiswerte Systeme, Plattformen und Trägersysteme- $\quad$ Angewandte Geographische Informationsverarbeitung XII - Agit 2006, Wichmann, Heidelberg

CAO LI, LI Hongbo, Kemper, G. (2008): Monitoring urban development of small Chinese cities using innovative aerial surveying technologies; Proceedings of the ISPRS Congress 2008 in Beijing

Kemper, G., Li Hongbo, Pauly, K. ${ }^{\dagger}$ (2008): New airborne Sensors and Platforms for specific applications in Photogrammetry and remote sensing; Proceedings of the ISPRS Congress 2008 in Beijing

Hine, D., Kemper, G., PivnickA, F., Li Hongbo (2008): Innovation in Flight Management Systems using real-time topological GIS Analysis; Proceedings of the ISPRS Congress 2008 in Beijing.

Vasel, R., Kemper, G., Schuhmann, R., Königer, F. (2008): New airborne Thermal Infrared photogrammetric applications and sensors for moisture detection; Proceedings of the ISPRS Congress 2008 in Beijing.

Barmettler, A., Nebiker, S., Kemper, G., Koch, C, Schmutz, M., Stutz, C., Peter, M., (2010) airAGro Fernerkundungslösung für die Agronomie auf der Basis von Leichtflugzeugen und Minidrohnen. 3 Ländertagung der DGPF, OVG und SGPBF Wien.

KEMPER, G. (2010): Neue luftgestützte Sensoren und Plattformen für verschiedenste Aufgaben in der Fernerkundung. 3 Ländertagung der DGPF, OVG und SGPBF, Wien. 